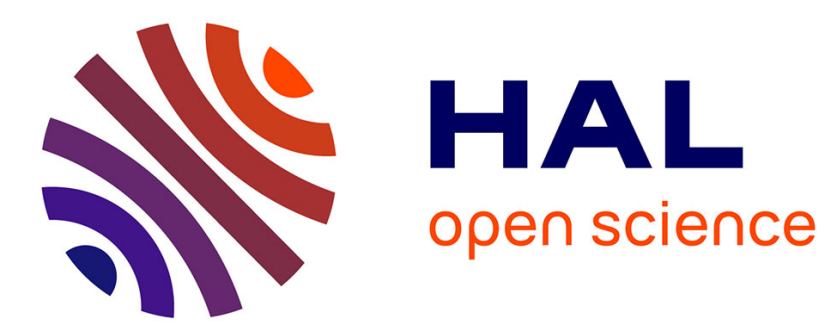

\title{
The influence of the learning data on the reduced order model of laminar non-premixed flames
}

Nicole Lopes Junqueira, Luis Fernando Figueira da Silva, Louise da Costa Ramos, Igor Braga de Paula

\section{- To cite this version:}

Nicole Lopes Junqueira, Luis Fernando Figueira da Silva, Louise da Costa Ramos, Igor Braga de Paula. The influence of the learning data on the reduced order model of laminar non-premixed flames. 26th International Congress of Mechanical Engineering, Nov 2021, Florianopolis, Brazil. 10.26678/ABCM.COBEM2021.COB2021-0110 . hal-03357849

\author{
HAL Id: hal-03357849 \\ https://hal.science/hal-03357849
}

Submitted on 1 Oct 2021

HAL is a multi-disciplinary open access archive for the deposit and dissemination of scientific research documents, whether they are published or not. The documents may come from teaching and research institutions in France or abroad, or from public or private research centers.
L'archive ouverte pluridisciplinaire $\mathbf{H A L}$, est destinée au dépôt et à la diffusion de documents scientifiques de niveau recherche, publiés ou non, émanant des établissements d'enseignement et de recherche français ou étrangers, des laboratoires publics ou privés. 


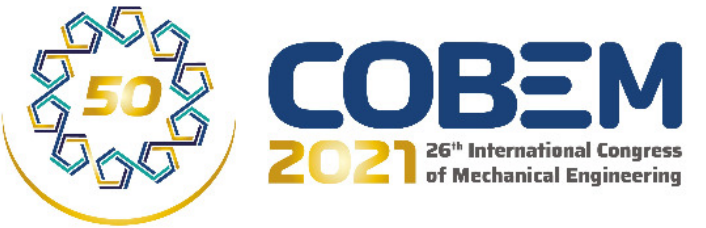

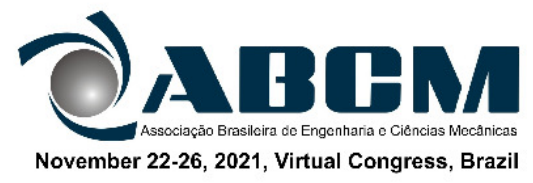

\section{COB-2021-0110 \\ The influence of the learning data on the reduced order model of laminar non-premixed flames}

\author{
Nicole Lopes Junqueira \\ Luís Fernando Figueira da Silva \\ Mechanical Engineering Department, Pontifícia Universidade Católica do Rio de Janeiro, Brazil \\ nicolelopes2@outlook.com; luifer@puc-rio.br

\section{Louise da Costa Ramos} \\ Ansys Fr., Centre Automatique et Systemes (CAS), Mines ParisTech, Paris, France \\ louise.ramos@ansys.com \\ Igor Braga de Paula \\ Mechanical Engineering Department, Pontifícia Universidade Católica do Rio de Janeiro, Brazil \\ igordepaula@puc-rio.br
}

Abstract. Computational fluid dynamics (CFD) is often applied to the study of combustion, enabling to optimize the process and control the emission of pollutants. This numerical methodology enables the analysis of different flame properties, such as the components of velocity, temperature, and mass fractions of chemical species. However, reproducing the behavior observed in engineering problems requires a high computational cost associated with memory and simulation time. Reduced order model (ROM) is a machine learning technique that has been applied to several engineering applications, aiming to develop models for complex systems with reduced computational cost. In this way, a high-fidelity model of complex systems is created from available data to learn its behavior and its main characteristics. In this work, different ROMs are created using CFD simulation data. The CFD model solves the mass, species, energy, and momentum conservation equations for a methane/air laminar diffusion flame, stabilized on the Gülder burner. Chemistry is modeled using a 19-species skeletal chemical kinetic mechanism. The static reduced order model uses the singular value decomposition (SVD) algorithm to decompose the CFD data and obtain the system's modes. Then, genetic aggregation response surface interpolation is applied on the higher SVD modes, creating the static ROM. This work analyzes the effect of different data preprocessing approaches on the ROM. The first analysis is the impact of reducing the number of learning data points, showing that this decrease does not directly impact the energy of the SVD modes, but, in the reconstruction field is possible to notice a degradation of the reconstruction. The second analysis is related to the effect of creating a ROM for each uncoupled flame property or treating the properties as a coupled system. The results of the coupled and uncoupled reduced order models are quite similar in terms of properties field reconstruction. However, in the energy analysis the coupled ROM converges rapidly, similarly to the uncoupled temperature ROM, while the uncoupled chemical species ROMs have a slower convergence.

Keywords: machine learning, computational fluid dynamics, diffusion flames, methane/air combustion

\section{INTRODUCTION}

Computational fluid dynamics (CFD) is widely used to analyze complex flow problems that are of interest to industry, such as combustion processes, because it enables the analysis of different flow properties. However, the high-fidelity calculation of engineering problems requires a significant computational burden associated with the simulation time and demanded memory (Kieckhefen et al., 2020). Reduced order model (ROM) is a technique that aims to represent highdimensional systems in a low-dimensional model, without compromise the accuracy and the efficiency of the model (Brunton et al., 2020; An et al., 2020). To construct a reduced model it is necessary to, first, collect a set of data from a system, where the variable parameters are the inputs of the model, then apply a reduction method and a machine learning algorithm (Da Costa Ramos et al., 2020). In the particular situations of combustion process, this input variable parameter might be an initial condition or a source term, any parameter that characterizes a property of the flow.

Regarding the data used in the learning process, machine learning approaches can be divided into two groups, online and offline. Online models learn the characteristics of the system at the same time the data is being collected, e.g., neural networks applied to image recognition (LeCun et al., 2015). Another example of this application is given by Pyta and Abel (2017), which estimated the eddy viscosity using an online identification, enabling the adaption of the reduced order 
model to the changes in the flow configuration. On the contrary, offline models learn the behavior based on previously collected data, using a decomposition algorithm to represent the sample data in reduced form, and an integration method to predict the result. For instance, Xiao et al. (2015) created a non-intrusive ROM of the Navier-Stokes equations of a flow passing a cylinder using a offline data collection.

Concerning the combustion process, machine learning techniques applications have been used for over a decade, as highlighted in the reviews of (Kalogirou, 2003; Ananthkrishnan et al., 2005). Chakravarthy et al. (2015) built a reduced order model using CFD simulations for oxy-coal combustion. Where was possible to quickly estimate the average outlet temperature of the burned gases for a given fuel and oxidant mass flow rates. And also determine the inlet mass flow rate to obtain the desired temperature. Wang et al. (2019) proposed a non-intrusive reduced order model using the POD-NN method, which combines the POD decomposition with a feed-forward neural network to construct a reduced order model to an unstable flow. Also using a non-intrusive methodology, Aversano et al. (2021)created a digital twin of a furnace was created, using CFD simulations and real-time measurements of an industrial system using the proper orthogonal decomposition and the Kriging method to find a response surface to an unexplored operation condition.

In this work, reduced-order models are constructed using CFD data of non premixed methane/air laminar flames, where the fuel inlet velocity is the variable input parameter. The objective is to analyze the influence of preprocessing the learning data on the results obtained by ROM. The first analysis is the influence of decreasing the amount of data provided to create the reduced model, comparing these results with those obtained by Junqueira et al. (2020). The second is the effect of building a ROM using the properties as a coupled system, i.e., using all the flow and combustion properties together as one only, instead of creating different models for each property as is done by Da Costa Ramos et al. (2020).

The combustion process is a multi-scale physics, which means that different processes occur at time scales spanning several orders of magnitude. Due to the diffusion present in flames, these time scales manifest as length scales (Law, 2006). However, the modeling of turbulent flames employing the methods developed for non-reacting turbulent flows ignores also this multi-scale nature of combustion by treating turbulence as a single-scale problem (Peters, 2009). This is why this work studies laminar flames, even though turbulent flames are more relevant to practical combustion processes. It is expected that a problem with multiple time and length scales will increase the complexity of the learning process.

The structure of this manuscript is as follows. First, the numerical model used to calculate the reactive flow is presented, outlining the hypothesis, chemical model, computational domain, boundary condition, solution methods, and mesh. Then, the steps to construct the reduced order model and the different ROM studied are given. Finally, the CFD and the ROM methodology results are discussed.

\section{METHODOLOGY}

In this section is presented the procedure used to model a non premixed flames, and the steps to construct reduced order model are described.

\subsection{Computational fluid dynamics}

The modeling of reactive flow involves the conservation of total mass, individual species, energy and momentum (Law, 2006), under the hypotheses of steady state, incompressible flow, ideal gas in the absence of gravity effects. The chemical reactions in methane/air flames, time scales are accounted for by using a skeletal mechanism (DRM19), a reduced kinetic based on GRI-Mech 1.2, to describe this mixture chemical pathways. This model contains 19 species plus $\mathrm{N}_{2}$ and Ar and 83 chemical reactions (Kazakov and Frenklach, 1984). Such mechanism was used to calculate a premixed methane/air flames in heated tubes and the Sandia flames (Gauthier et al., 2012; Labahn et al., 2017). The species transport equations considers the Soret thermal diffusion effect and the diffusion energy source. The mixture properties are considered incompressible ideal gas for the density; mixing law is applied for the specific heat; ideal gas mixing law for thermal conductivity, viscosity; and kinetic theory for the mass diffusivity and the thermal diffusion coefficient.

The finite volume numerical model of the laminar non premixed flame stabilized on the Gülder burner (De Castro and Figueira da Silva, 2019) was created using the Ansys software Fluent 2019 R3 (Junqueira et al., 2020). So, it is important to define, first, the geometry and the mesh. Then, is outlined the numerical simulation setup, such as the boundary conditions and the calculation methods.

The studied Gülder burner presents an axial symmetry flow and it has two inlets, as shown in Fig. 1 (Jerez et al., 2019). The air enters through an annular region with a radius of $50 \mathrm{~mm}$, and the fuel enters through the central tube, which the diameter is $11 \mathrm{~mm}$. Since the studied flame is axisymmetric, a two dimensional geometry is used. The domain is divided into two parts allowing different mesh refinements along the domain. The internal part (A) is composed of the central tube, where there is only fuel flow, and the estimated mixture region, where the chemical reactions are supposed to occur. The external part (B) includes only the air entrance and the outlet, in other words, only air and burned gases flows.

Regarding the boundary conditions, Fig. 1 presents a schematic domain of the boundary conditions using different colors to represent them. The axis of symmetry is the $160 \mathrm{~mm}$ yellow line; the four grey lines are the walls, the outlet and the two inlets are represented by the red line and the blue lines, respectively. There are two category of walls in this domain; 
the burner wall, which has an adiabatic and a no-slip condition, and the one representing the outer boundary, which has a slip and constant temperature condition. The walls between the burner inlets are denominated as burner walls, and the free boundary is the external right boundary, with a length of $150 \mathrm{~mm}$. Concerning the inlets, it is specified the species molar fraction, the inlet velocity and temperature. Following (Junqueira et al., 2020), the air prescribed inlet velocity is uniform, $v_{a, i}=60 \mathrm{~cm} / \mathrm{s}$ and the fuel prescribed inlet velocity $\left(v_{f, i}\right)$ is uniform and varies between 1.75 and $4.38 \mathrm{~cm} / \mathrm{s}$. In this work, the air is composed by $21 \% \mathrm{O}_{2}$ and $79 \% \mathrm{~N}_{2}$ and the fuel is methane. Air and fuel have prescribed temperature and pressure of $300 \mathrm{~K}$ and $1 \mathrm{~atm}$, respectively.

This multi-scale problem uses the following solution methods: coupled for the pressure-velocity coupling, Presto for the spatial discretization of pressure, and the second order upwind to calculate the spatial discretization of energy, species and momentum. The method used to calculate the reaction rate of the species transport equation is the stiff chemistry solver with the ISAT table as integration method and with an error tolerance of $10^{-4}$.

To properly describe the chemical reaction length scales, a refined mesh is necessary, and this is one of the reasons why the domain is divided into two parts. Therefore, to reduce the computational burden, it is adopted different mesh size along the domain and a mesh adaptation is applied. Since the internal part (A) is the estimated reactive zone, it has a more refined mesh. The external part (B) has a uniform mesh with a size of $1 \mathrm{~mm}$. The internal part has a rectangular mesh, with a size of $100 \mu \mathrm{m}$ and $1 \mathrm{~mm}$ in the radial and axial directions, respectively. Mesh adaptation is used to refine the mesh in specific regions. In this work, a gradient mesh adaptation based on the temperature with a refinement and coarsening thresholds of $10 \mathrm{~K} / \mathrm{m}$ and $300 \mathrm{~K} / \mathrm{m}$, respectively. The mesh adaptation tool is applied every 25 iterations, and in the end a mesh of 93,168 nodes is obtained, 81501 nodes more than the original mesh.

\subsection{Reduced order model}

Reduced order model (ROM) is a machine learning technique that enables to decrease the computational cost of describing complex systems of high dimension, preserving the main characteristics associated to it, while controlling the accuracy and efficiency of the model (Brunton et al., 2020). This technique has been applied to different engineering problems (Zhao, 2021; Aversano et al., 2021). In this work, the software Static ROM from Twin Builder is used to construct the reduced order models.

The construction of the ROM begins with the division of the sample data into two groups; the learning data, which is used to build the model, and the validation data, used to assess the error. Typically $60 \%$ of the dataset is used as learning data, and the remaining $40 \%$ as validation data. The division of the dataset is done randomly, however, to avoid extrapolation of the reduced model, the parametric variable limits must be included among the learning data. Note that the data set consists of different cases, where each case contains the values of a computed property as a function of the variable property.

After setting the learning and the validation data, the learning data is organized into a matrix, where the number of columns equals the number of cases, and the number of rows equals the number of mesh points in the CFD simulation. The singular value decomposition (SVD) algorithm is used to decompose this matrix (Trefethen, 1997). Then, the first singular modes are retained, as these contain the most important characteristics of the system, and thus this is the reduced base of the learning data that describes the complex system.

The last step of the ROM is the interpolation of the modes. The interpolation method used is the Genetic Aggregation Response Surface (GARS) which is a meta model constructed using four different integration methods; polynomial regression, Kriging, support vector regression and moving least squares (Ostertagová, 2012; Kaymaz, 2005; Smola and Schölkopf, 2004; Lancaster and Salkauskas, 1981; ANSYS, 2020). The GARS uses a selection process to determine the combination of response surface (RS) that best describes the system (Viana et al., 2009; Ben Salem et al., 2017). Ben Salem and Tomaso (2018) proposed a surrogate model selection algorithm based on the Genetic Aggregation using a penalized predictive score (PPS), i.e. 


$$
\widehat{A}_{m}(x)=\sum_{l=1}^{m} \omega_{l} \hat{s}^{(l)}(x)
$$

where, $\widehat{A}_{m}$ represents the aggregation of the $m$ surrogates models $(\hat{s})$ weighed by $\omega_{l}$, which is calculated using the PPS method (Ben Salem and Tomaso, 2018). The genetic aggregation algorithm is applied in the reduced base to create a model that describes the behavior of the system for an arbitrary value of the variable parameter. Then, the prediction of the validation data is performed.

\subsection{ROM construction specifics}

Here, the nomenclature chosen to classify the reduced order models studied summarizes the main information about the learning data. It states the number of learning cases, the number of modes used, and whether the ROM has uncoupled or coupled combustion properties.

$$
\operatorname{ROM}_{(i, j)}^{(c|w| p)}
$$

the subscript $(i, j)$ refers to the number of learning cases and the number of modes, respectively; and the superscript $(c|w| p)$ refers to the data. For instance, if the combustion properties are treated as uncoupled $(u)$ or coupled $(c)$, if any normalization is applied $(n)$ or not $(w)$, and the specific property studied for example: $v_{x}$ and $v_{y}$ for the axial and radial velocity or $T$ for the temperature. In the case of the coupled ROM, different nomenclature is used $(p c, p s)$. Meaning that first is presented the properties that are coupled, and then the property of interest.

Since the amount of data is related to the accuracy of the model, the first analysis focus on the effect of the quantity of data $(i)$ on the learning set. To this end, two ROM are created, with the same number of modes $(j)$. The second effect studied is the properties coupling. Indeed, the properties of the flame are closely coupled via the chemical reaction. Since the non-premixed flame studied is multi-scale, normalization of temperature and the velocity components effects are investigated.

$$
T^{*}=\frac{T(k)-T_{0}}{T_{a d}-T_{0}}, \quad u^{*}=\frac{u(k)}{u_{\max }}, \quad u_{\max }=u_{0} \frac{T_{a d}}{T_{0}}
$$

Where $T^{*}$ and $u^{*}$ are the normalized temperature, and the normalized velocity component; $T_{0}$ is the temperature of fresh gas, $300 \mathrm{~K} ; T_{a d}$ is the adiabatic flame temperature of stoichiometric methane/air mixtures, $2236 \mathrm{~K}$, and $u_{0}$ is the air inlet velocity, $60 \mathrm{~cm} / \mathrm{s}$. It is interesting to highlight that it is possible to estimate the temperature and velocity bounds in the reactive case. Indeed, the reference temperature in the chemical equilibrium $\left(T_{a d}\right)$ and the velocity are known beforehand. However, each chemical species concentration is unknown a priory, so it is more complicated to estimate a reference. Thus, to understand the influence of normalization, a reduced order model using the properties coupled without the normalization and with the normalization of temperature and velocity are studied.

\section{RESULTS AND DISCUSSION}

This section presents and analyzes the results obtained through the computational fluids dynamics and reduced order model. First is presented the flame structure for the lower and higher fuel inlet velocities studied. Then, the influence of decreasing the number of learning data and the effects of coupling the properties to create the ROM is analyzed.

\subsection{Flame structure}

In order to discuss the flame structure and the influence of the prescribed fuel inlet velocity, Fig. 2 shows a qualitative comparison of the classic flame structure at the extremes of the studied velocity range. The first figures (2a, 2b and 2c) present the velocity components and the temperature, whereas the remaining figures $(2 \mathrm{~d}, 2 \mathrm{f}$ and $2 \mathrm{e})$ reproduce the mass fraction field of three chemical species characteristic of the methane/air combustion, i.e., $\mathrm{OH}, \mathrm{CH}_{2}$ and $\mathrm{CO}$. The axial and radial components of the velocity field are shown in Figs. $2 a$ and $2 b$, respectively. Note that, along the flame, the axial component of the velocity increases as it gets further from the fuel tube exit. This behavior is explained by the increasing temperature, which affects the density within the reaction zone, and by the conservation of the total mass equation and the hypothesis of ideal gas: as the temperature increases the velocity increases. As for the radial component, most of the domain, fuel inlet tube and the regions far from the flame front, have zero velocity for both cases as expected. However, when comparing the two cases, the region of positive radial velocity is larger for the case of $v_{f, i}=4.38 \mathrm{~cm} / \mathrm{s}$. 
The temperature profile, Fig. 2c, shows the high temperature region, i.e., the flame region. The maximum temperature is $2,138 \mathrm{~K}$ for the slowest fuel inlet velocity, and for the largest velocity is $2,153 \mathrm{~K}$, both are close to the adiabatic flame temperature for methane $(2,236 \mathrm{~K})$. Downstream the maximum temperature, the usual temperature decrease is observed along the $y$ axis, due to the combustion products mixture with air, which is at $300 \mathrm{~K}$. As expected, the velocity has a significant influence on the flame, and this is seen in the temperature profiles; as the fuel input velocity increases, the flame becomes longer. It is worth noticing that, for the lowest fuel velocity case, there is a region within the fuel tube that has a temperature greater than $300 \mathrm{~K}$, which causes the preheating of fuel.

Figures $2 \mathrm{~d}, 2 \mathrm{e}$ and $2 \mathrm{f}$ show the mass fraction fields of the chemical species: hydroxyl $(\mathrm{OH})$, carbon monoxide $(\mathrm{CO})$ and methylene $\left(\mathrm{CH}_{2}\right)$, respectively. The position and shape of the flame are often estimated using the $\mathrm{OH}$ mass fraction profile. The highest concentration of $\mathrm{OH}$ is present near the tube walls for both inlet velocity values. However, for the higher fuel velocity, the region of maximum $\mathrm{OH}$ concentration and the flame height are longer. Carbon monoxide is present within the flame region also, and for the case of lower fuel velocity $\mathrm{CO}$ is found within the fuel inlet tube. This is associated to the increase in fuel temperature inside the tube, seen in Fig. 2c, which is related to the shorter flame length due to the smaller fuel flow rate. It is worth to stress that for the higher velocity case, the region of maximum concentration of this chemical species is wider than the region found for the lower fuel velocity case.

Examining the mass fraction field of $\mathrm{CH}_{2}$, Fig. 2e, it is observed that the concentration of this species is of the order of $10 \mathrm{ppm}$ and that the concentration region of $\mathrm{CH}_{2}$ is quite narrow (approximately $0.5 \mathrm{~mm}$ ). It is important to highlight that this species is the one with the thinnest scale, since it is quickly created and consumed inside the reactive region, and this is an effective indicator of the flame front (Da Costa Ramos et al., 2020). The maximum concentration is located near the burner wall, and for the lowest fuel inlet velocity case is $29 \mathrm{ppm}$. This property was used as the convergence parameter, i.e., when the field of the mass fraction of $\mathrm{CH}_{2}$ stops varying, all other variables are assumed as converged.

It should be stressed that each CFD computation required around 15 days to reach convergence, using 18 cores of a 24 CPU in a computer with 32 GB of memory using windows 10 .

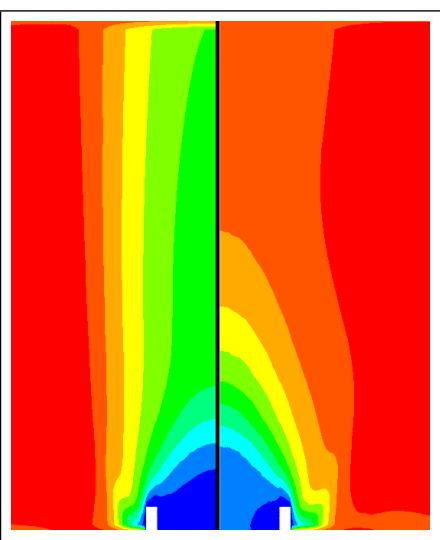

(a) Axial velocity component $\in(0,67.7)[\mathrm{cm} / \mathrm{s}]$.

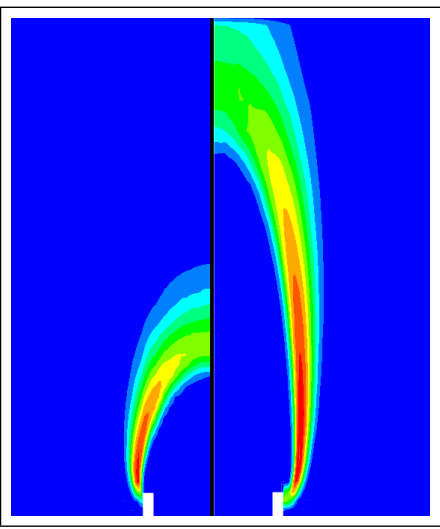

(d) $\mathrm{OH}$ mass fraction $\in(0,3.89 e-3)$.

Figure 2. Comparison of the flame structures of $(-17.2,17.2)[\mathrm{cm} / \mathrm{s}]$.

(e) $\mathrm{CH}_{2}$

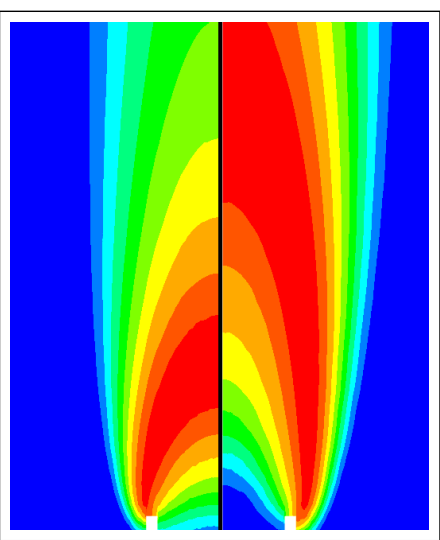

(b) Radial velocity component $\epsilon$

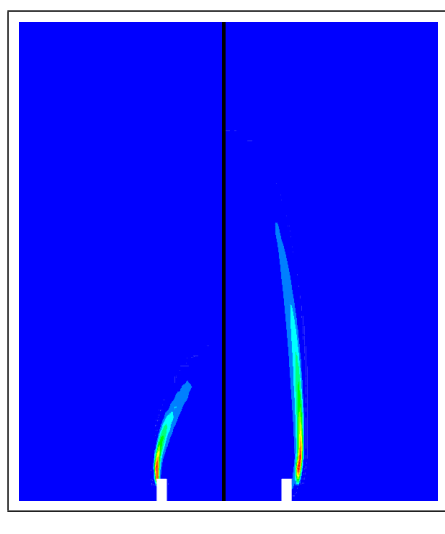
mass

fraction $\in(0,2.67 e-5)$ (c) $\in(300,2158)[\mathrm{K}]$.

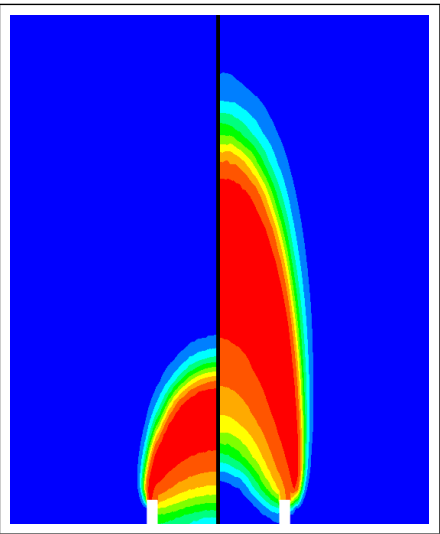

(f) $\mathrm{CO}$ mass fraction $\in(0,5.11 e-2)$. $v_{f, i}=4.38 \mathrm{~cm} / \mathrm{s}$, (right) prescribed fuel inlet velocity. The color map goes from blue (minimum) to red (maximum). 


\subsection{Number of learning cases influence}

Since each CFD computation is time consuming, but the ROM generation typically requires 1 minute only, the first analysis performed concerns the number of learning cases that influence the ROM features. To this end, first, is analyzed for the uncoupled properties ROM, without resorting to normalization, the energy distribution in each of the SVD modes for two reduced order models, one using 4 learning cases $\left(R O M_{(4,3)}^{(u|w| p)}\right)$, and the other, using 12 learning cases $\left(\operatorname{ROM}_{(12,3)}^{(u|w| p)}\right)$. Then, a comparison of the results obtained through CFD and the reduced order models, via a qualitative analysis, of six properties: axial and radial velocity components; temperature; $\mathrm{OH}, \mathrm{CH}_{2}$ and $\mathrm{CO}$ mass fraction.

Therefore, the energy required to reconstruct the decomposed matrix can be characterized by the singular values. Figure 3 shows the singular values normalized by their sum as a function of the number of modes for each property analyzed in this work. As expected from the SVD theory, all the flame properties have the same behavior, the highest amount of energy is located in the first mode, and then this energy decreases as the number of modes increases. Further more, the energy distribution for $\operatorname{ROM}_{(4,3)}^{(u|w| p)}$ and $R O M_{(12,3)}^{(u|w| p)}$ are similar, and the number of learning cases exerts a small influence on the energy for a given the number of modes. Indeed, for the components of velocity and the temperature, the first mode has more than $80 \%$ of energy, and the second mode decreases to less than $15 \%$. In contrast, for the species, the first modes has less than $60 \%$ of energy, and the second and third modes has energy around $20 \%$ and $10 \%$, respectively. So, it is easier for the ROM to learn the behavior of the temperature and velocity components than the chemical species, since its first modes contains more information. It is important to notice that, with the notable exception of the ROM for the $\mathrm{CH}_{2}$ mass fraction, the fourth mode contains less than $10 \%$ of energy for all species.

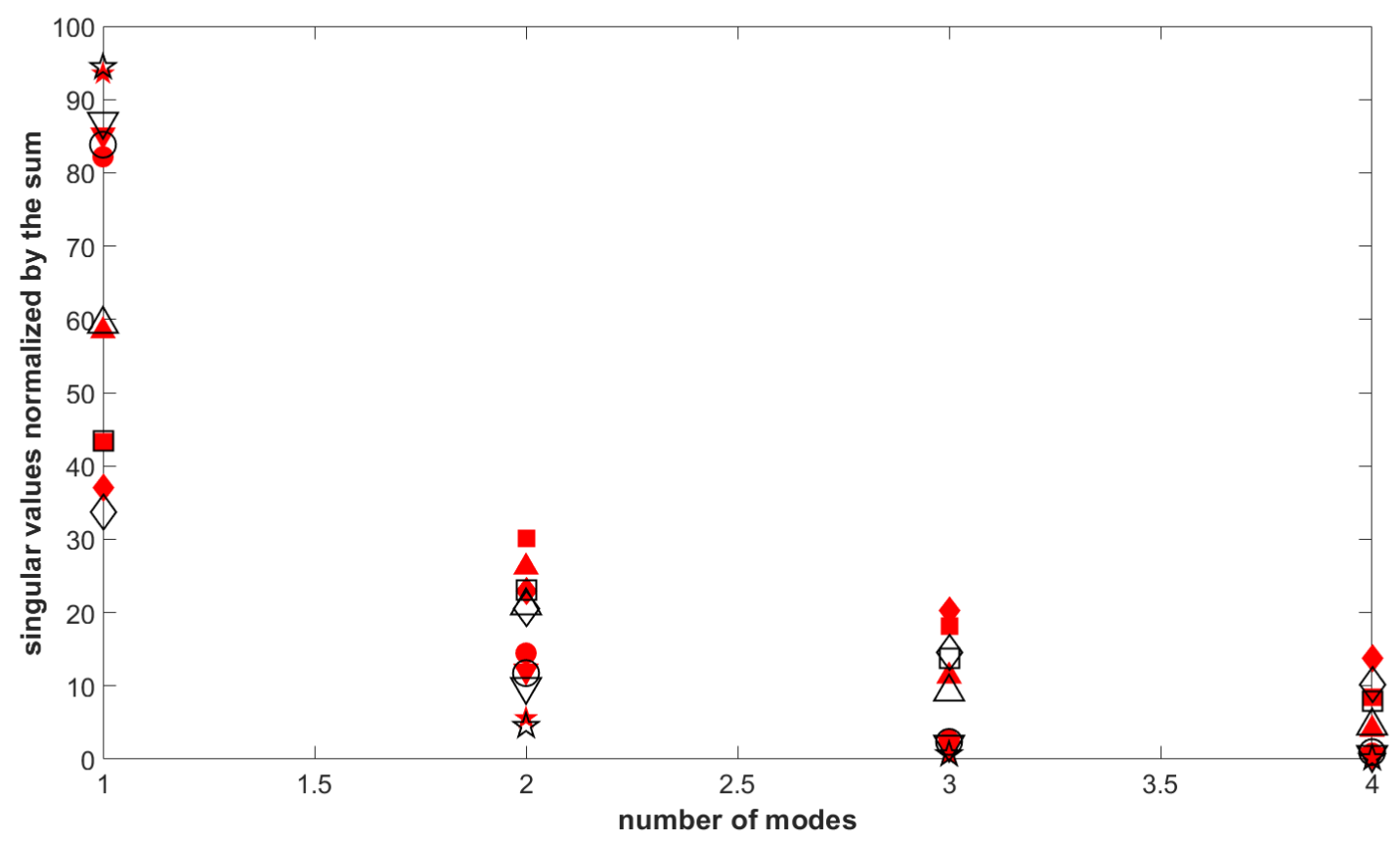

Figure 3: Singular values normalized by the sum $\times$ number of modes. Filled symbols: ROM using 4 learning cases; nonfilled symbols: ROM using 12 learning cases. $\nabla$ : temperature; $\star$ : axial velocity component; $\circ$ : radial velocity component; $\square: \mathrm{OH}$ mass fraction; $\diamond: \mathrm{CH}_{2}$ mass fraction; $\triangle$ : $\mathrm{CO}$ mass fraction.

To characterize the influence of decreasing the number of cases used to learn the model, Fig. 4 exhibits a qualitative comparison of the computational fluids dynamics results ( $\mathrm{a}, \mathrm{d}, \mathrm{g}, \mathrm{j}, \mathrm{m}$ and $\mathrm{p}$ ), the reduced order models with 12 learning cases (b, e, h, k, n and q) and with 4 learning cases (c, f, i, 1, o and r). It is noticed that the results for the axial and radial components are quite similar for both reduced order models. The axial velocity (Fig. 4a, 4b and 4c) presents a slower velocity inside the fuel inlet tube and a higher velocity near the external wall and the air inlet. However, for the $\operatorname{ROM}_{(4,3)}^{\left(u|w| v_{x}\right)}$ (Fig. 4c) a region with a velocity higher than $67.7 \mathrm{~cm} / \mathrm{s}$ is observed in white. On the other hand, the radial velocity (Fig. 4d, 4e and 4f) profiles are almost identical, in particular, both results present most of the domain with zero velocity, a positive area near the fuel outlet and a small negative area in the air region.

Concerning the temperature fields (Fig. $4 \mathrm{~g}, 4 \mathrm{~h}$ and $4 \mathrm{i}) R O M_{(4,3)}^{(u|w| T)}$ exhibits a region of maximum temperature that is smaller than that calculated with CFD and, also, the predicted by the $R O M_{(12,3)}^{(u|w| T)}$. Indeed, the maximum temperature 
predicted by the $R O M_{(12,3)}^{(u|w| T)}$ is $2091 \mathrm{~K}$, and for the $R O M_{(4,3)}^{(u|w| T)} 2016 \mathrm{~K}$, which correspond to errors of $50 \mathrm{~K}$ and $125 \mathrm{~K}$, respectively, when compared with the CFD calculated maximum temperature $(2141 \mathrm{~K})$.

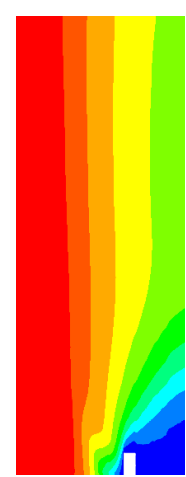

(a) CFD $v_{x}$.

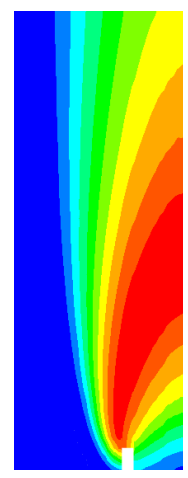

(g) CFD $T$

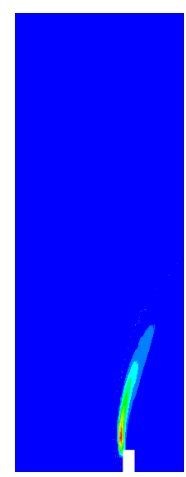

(m) CFD

$\mathrm{CH}_{2}$.

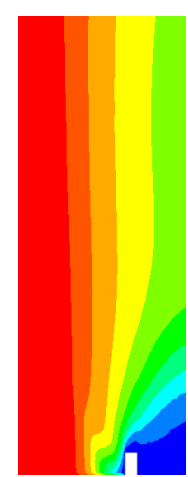

(b)

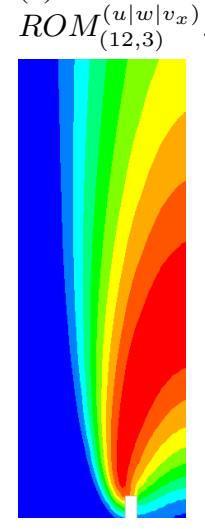

(h)

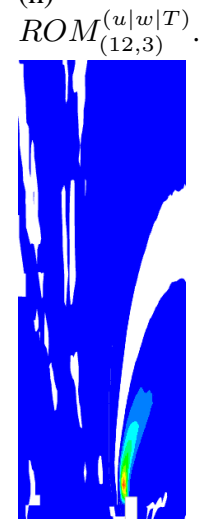

(n)

$\operatorname{ROM}_{(12,3)}^{(u|w| C H 2)}$

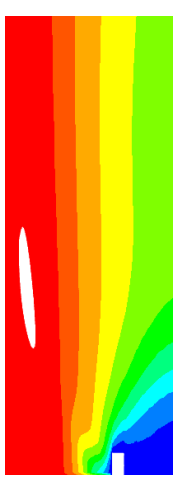

(c)

$\operatorname{ROM}_{(4,3)}^{\left(u|w| v_{x}\right)}$.

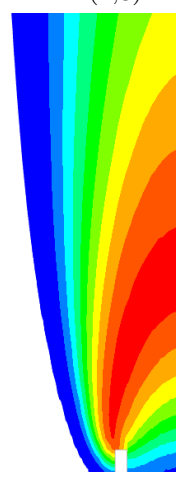

(i)

$R O M^{(u|w| T)}$

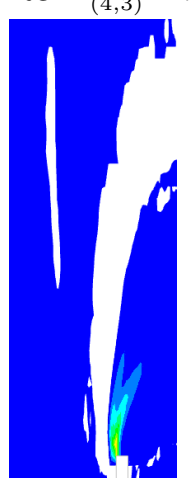

(o)

$R O M_{(4,3)}^{(u|w| C H 2)}$.

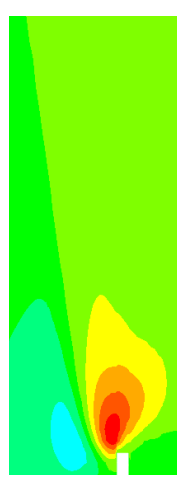

(d) CFD $v_{y}$.

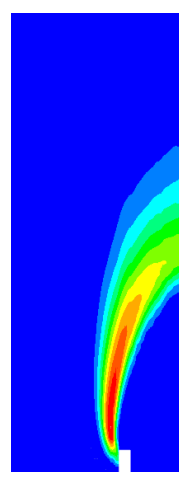

(j) $\mathrm{CFD} \mathrm{OH}$.

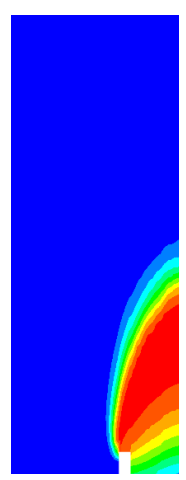

(p) CFD CO.

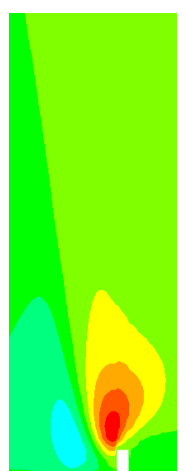

(e)

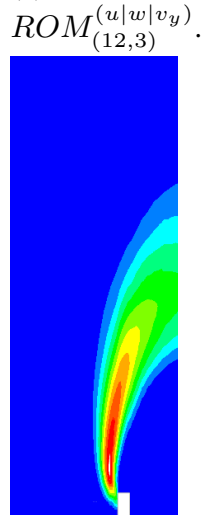

(k)

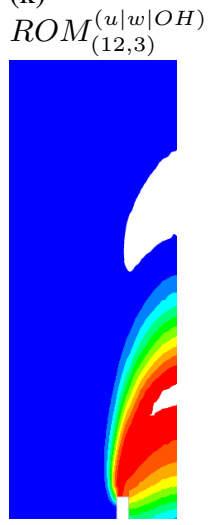

(q)

$R O M_{(12,3)}^{(u|w| C O)}$.

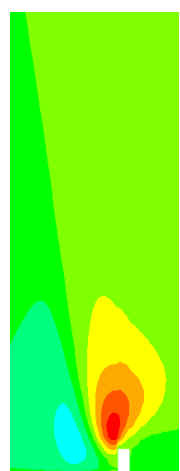

(f)

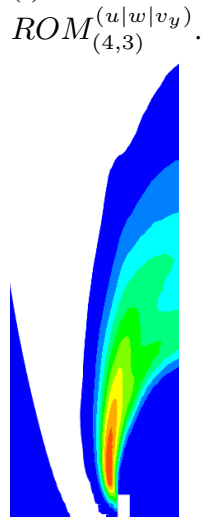

(1) $R O M^{(u|w| O H)}$

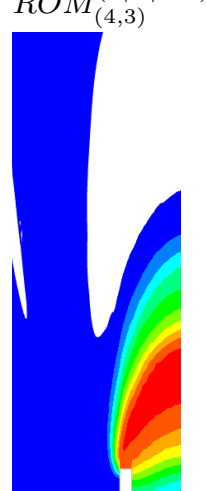

(r) $R O M_{(4,3)}^{(u|w| C O)}$.

Figure 4: Comparison of the results obtained with computational fluid dynamics (CFD), and reduced order model using 12 learning cases $\left(\operatorname{ROM}_{(12,3)}^{(u|w| p)}\right)$ and 4 learning cases $\left(R O M_{(4,3)}^{(u|w| p)}\right)$, for the validation case with prescribed fuel inlet velocity of $v_{f, i}=2.11 \mathrm{~cm} / \mathrm{s}$. The axial component of the velocity (a,b and c) varies between 0 to $64.6 \mathrm{~cm} / \mathrm{s}$; the radial component of velocity $(\mathrm{d}$, e and $\mathrm{f}$ ) varies $(-16,16) \mathrm{cm} / \mathrm{s}$; the temperature $(\mathrm{g}$, h and i) varies between $(300,2,141) \mathrm{K}$. The $\mathrm{OH}$ mass fraction ( $\mathrm{j}, \mathrm{k}$ and $\mathrm{l}$ ) varies between 0 to $3.71 \cdot 10-3$; the $\mathrm{CH}_{2}$ mass fraction ( $\mathrm{m}, \mathrm{n}$ and o) varies $[0,2.83 \cdot 10-5]$; the $\mathrm{CO}$ mass fraction ( $\mathrm{p}, \mathrm{q}$ and $\mathrm{r}$ ) varies between $[0,5.1 \cdot 10-2]$. The color map goes from blue (minimum) to red (maximum). The fuel inlet is located in the bottom right side, and in the left side is the air inlet.

The $\mathrm{OH}$ mass fraction (Fig. $4 \mathrm{j}, 4 \mathrm{k}$ and $4 \mathrm{l}$ ) results also show that upon increasing the number of learning cases, the reconstruction error decreases, and thus the description of the flame front improves from $R O M_{(4,3)}^{(u|w| O H)}$ to $R O M_{(12,3)}^{(u|w| O H)}$, becoming more similar to the CFD result. Thus, it is possible to notice that in the $R O M_{(12,3)}^{(u|w| O H)}$, the $\mathrm{OH}$ mass fraction is not as spread as the $\operatorname{ROM}_{(4,3)}^{(u|w| O H)}$ result. Another important aspect is that the ROM results exhibit a region with negative mass fraction, but with different order of magnitude, i.e., for the $R O M_{(4,3)}^{(u|w| O H)}$ the minimum value is $-6 \cdot 10^{-5}$ whereas 
for the $\operatorname{ROM}_{(12,3)}^{(u|w| O H)}$ it is $-3 \cdot 10^{-13}$.

The reconstructed $\mathrm{CH}_{2}$ mass fraction fields also present regions with negative values in both ROM results as may be seen in Fig. 4n and 4o. Furthermore, in accordance with the CFD result, the $\operatorname{ROM}_{(12,3)}^{(u|w| C H 2)}$ has a longer profile and a less spread than the reduced order model with 4 learning cases. Regarding the maximum mass fraction, both ROM led to values smaller than the calculated with CFD $\left(2.83 \cdot 10^{-5}\right), 2.54 \cdot 10^{-5}$ and $2.03 \cdot 10^{-5}$ for the $R O M_{(12,3)}^{(u|w| C H 2)}$ and $R O M_{(4,3)}^{(u|w| C H 2)}$, respectively.

Concerning now the mass fraction of $\mathrm{CO}$, depicted at Figs. $4 \mathrm{p}, 4 \mathrm{q}$ and $4 \mathrm{r}$, it is seen that the maximum concentration region is smaller for $\operatorname{ROM}_{(4,3)}^{(u|w| C O)}$ than the CFD field, but both ROMs show a longer CO region than that calculated by CFD, in particular downstream the flame front. Furthermore, for $R O M_{(12,3)}^{(u|w| C O)}$, the maximum CO concentration is 0.0516, whereas the CFD value is 0.0510 . It is worth noting that both reconstruction exhibit a negative mass fraction region in the domain, but $R O M_{(12,3)}^{(u|w| C O)}$ presents smaller regions.

\subsection{Coupled properties}

The results discussed in the preceding section establish that 12 learning cases and three modes lead to a ROM that better describe the CFD data when the properties are reconstructed as uncoupled fields. In order to assess the effect of coupling, the comparison of the energy for the reduced order model with the properties treated uncoupled $\left(R O M_{(12,5)}^{(u|w| p)}\right)$ and treated as coupled $\left(\operatorname{ROM}_{(12,5)}^{(c|w / n| a l l)}\right)$ is given in Fig. 5. This figure depicts two cases of coupled properties, one without normalization $(w)$, and the other with temperature and velocity normalized using Eq. 3. The results show that the energy required to reconstruct the ROM using the properties coupled without a normalization is similar to that of the temperature ROM. This occurs because the singular values are controlled by the higher absolute value property, in this case, the temperature. Despite the normalization, the energy content is similar to that required to reconstruct the temperature. A possible reason for that is the fact that some species have small concentrations, such as $\mathrm{CH}_{2}$ or $\mathrm{OH}$, which vary between the order of magnitude $\left(1 \cdot 10^{-6}, 1 \cdot 10^{-3}\right)$ whereas the temperature varies between $(0,1)$.

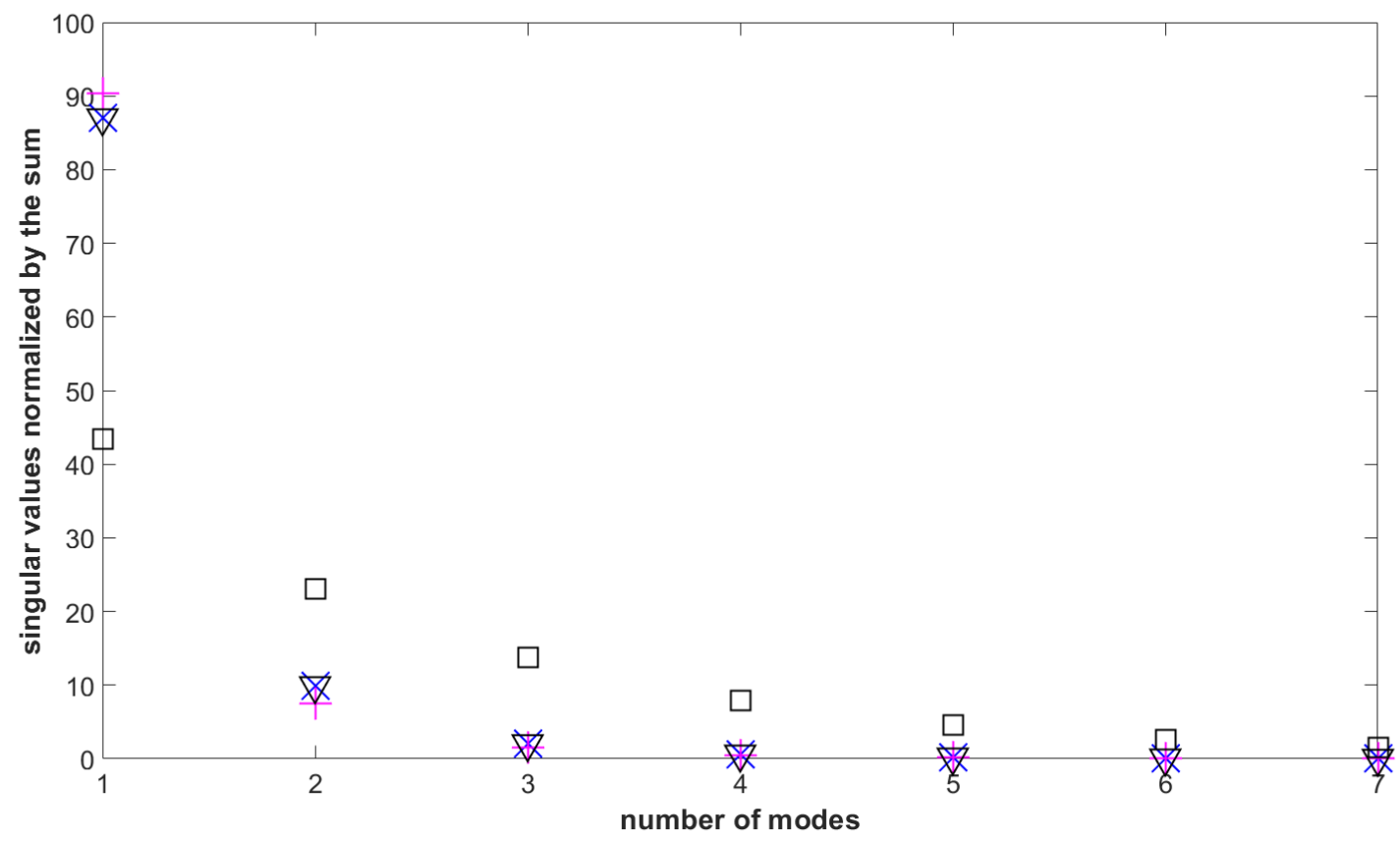

Figure 5: Singular value normalized by the sum $\times$ number of modes. $\nabla$ : temperature; $\square:$ OH mass fraction; $\times$ : Properties treated as coupled, without normalization; +: Properties treated as coupled, with temperature and velocity components normalized.

The three reduced order model compared here are reconstructed using five modes and 12 learning cases. Since the number of modes is chosen based on the energy (Fig. 5), and after the sixth mode, the energy varies very slightly, each mode having a value smaller than $0.1 \%$. Due to that, the energy does not decrease more, and then, five modes is considered as the optimum number of modes to reconstruct the problem. The first is the uncoupled ROM, where the data of one property is used separately to reconstruct the model $\left(R O M_{(12,5)}^{(u|w| p)}\right)$. The other ROM uses the 22 properties 
coupled (temperature, velocity components, and 19 chemical species).

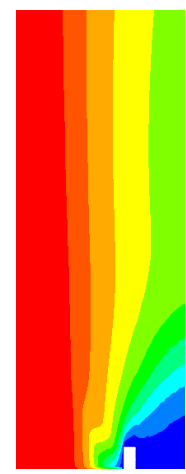

(a)
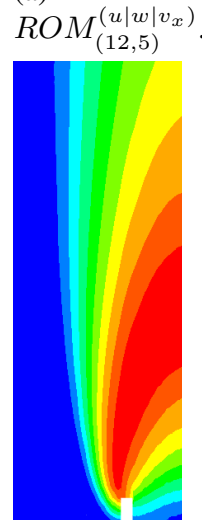

(g)

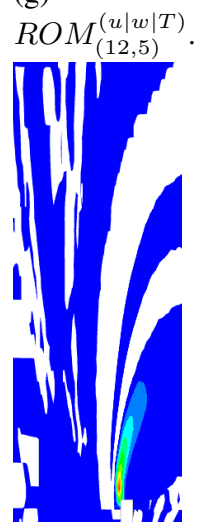

$(\mathrm{m})$

$R O M_{(12,5)}^{(u|w| C H 2)}$

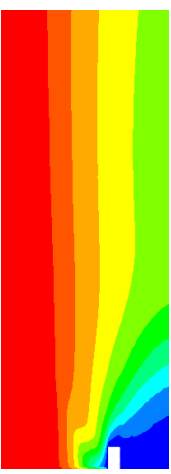

(b)
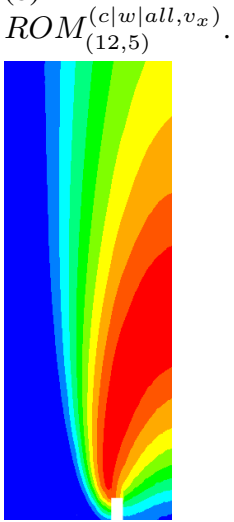

(h)
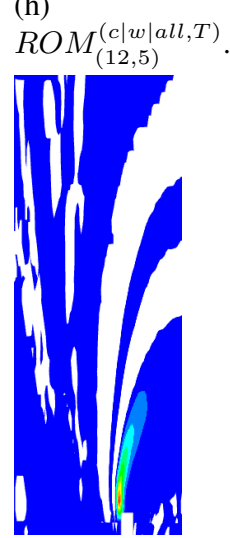

(n)

$R O M_{(12,5)}^{(c|w| a l l, C H 2)}$.

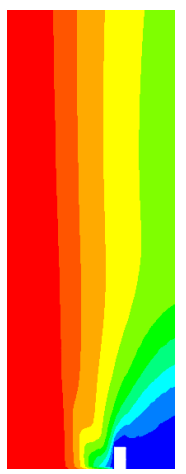

(c)
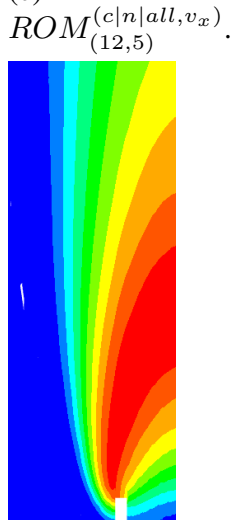

(i)

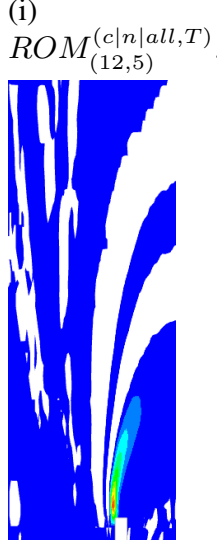

(o)
$R O M_{(12,5)}^{(c|n| a l l, C H 2)}$

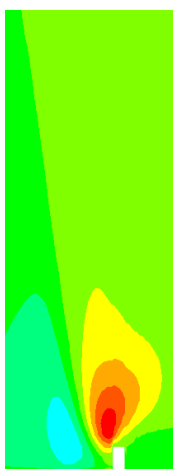

(d)
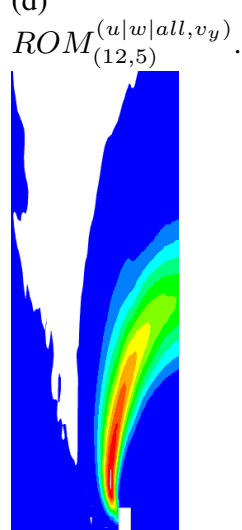

(j)

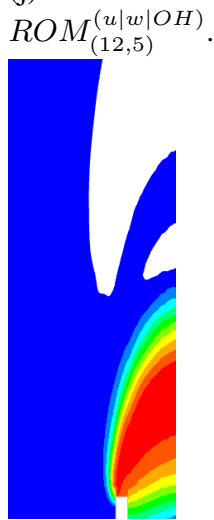

(p)

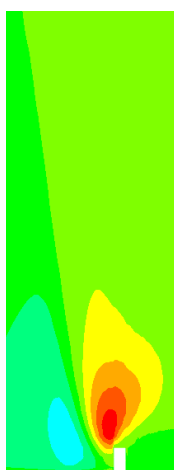

(e)
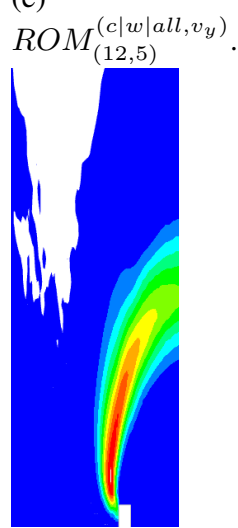

(k)

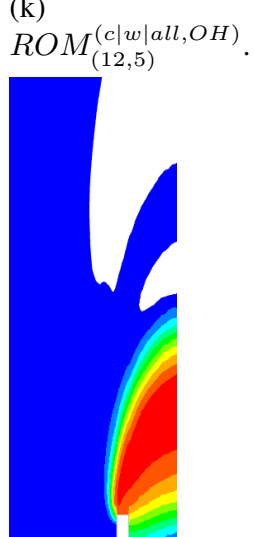

(q)

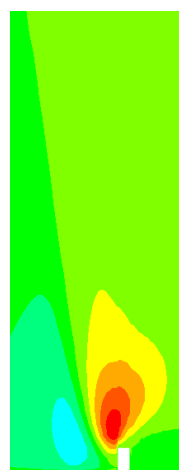

(f) $\operatorname{ROM}_{(12,5)}^{\left(c|n| a l l, v_{y}\right)}$

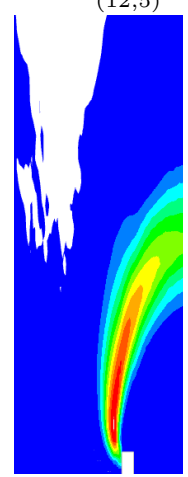

(1) $R O M_{(12,5)}^{(c|n| a l l, O H)}$

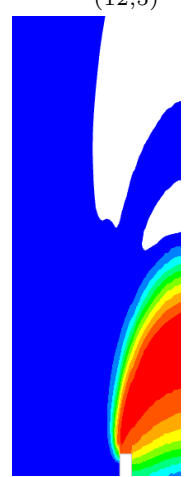

(r) $R O M_{(12,5)}^{(c|n| a l l)}$

Figure 6: Comparison of the results obtained with reduced order model using 5 modes for the properties treated uncoupled $\left(\operatorname{ROM}_{(12,5)}^{(u|w| p)}\right)$, coupled without normalization $\left(\operatorname{ROM}_{(12,5)}^{(c|w| a l l)}\right)$ and coupled with the temperature and components of velocity normalized $\left(R O M_{(12,5)}^{(c|n| a l l)}\right)$; for the validation case with prescribed fuel inlet velocity of $v_{f, i}=2.11 \mathrm{~cm} / \mathrm{s}$. The axial component of the velocity ( $\mathrm{a}, \mathrm{b}$ and $\mathrm{c}$ ) varies between 0 to $64.6 \mathrm{~cm} / \mathrm{s}$; the radial component of velocity (d, e and $\mathrm{f}$ ) varies $(-16,16) \mathrm{cm} / \mathrm{s}$; the temperature $(\mathrm{g}, \mathrm{h}$ and $\mathrm{i})$ varies between $(300,2,141) \mathrm{K}$. The $\mathrm{OH}$ mass fraction $(\mathrm{j}, \mathrm{k}$ and $\mathrm{l})$ varies between 0 to $3.71 \cdot 10^{-3}$; the $\mathrm{CH}_{2}$ mass fraction $\left(\mathrm{m}, \mathrm{n}\right.$ and o) varies $\left[0,2.83 \cdot 10^{-5}\right]$; the $\mathrm{CO}$ mass fraction (p, $\mathrm{q}$ and $\mathrm{r}$ ) varies between $\left[0,5.1 \cdot 10^{-2}\right]$. The color map goes from blue (minimum) to red (maximum). The fuel inlet is located in the bottom right side, and in the left side is the air inlet.

Explaining first the axial and radial velocity components, given in Fig. 6a-6f the ROM results are similar, and with the same maximum for the axial velocity, $67.7 \mathrm{~cm} / \mathrm{s}$. It is also interesting to observe that $R O M_{(12,5)}^{(u|w| p)}$ and $R O M_{(4,3)}^{(u|w| p)}$ (Fig. 6a and 4b; Fig. 6d and 4e) results are also very similar, which means that decreasing the number of modes does not significantly influences the reconstructed velocity components. The reconstructed temperature, with the properties coupled, normalized or non-normalized are nearly identical exhibiting the same maximum temperature (2,148 K). However, the normalized ROM result (Fig. 6i) presents a small white area, which means that the temperature is slightly smaller 
than $300 \mathrm{~K}$. Comparing these results, the $R O M_{(12,3)}^{(u|w| T)}$, Fig. 4h, and the CFD result (Fig. 2c) shows that the maximum temperature is closer to that calculated by CFD than that predicted by the ROM with 3 modes, but it slightly exceeds $7 \mathrm{~K}$ of the one calculated.

Regarding the species $\mathrm{OH}, \mathrm{CH}_{2}$ and $\mathrm{CO}$ mass fraction (Fig. 6j-6r), all the results for $R O M_{(12,5)}^{(u|w| s)}$ and $R O M_{(12,5)}^{(c|w| a l l, s)}$ resemble each other quite closely, with the maximum concentration varying very slightly between them. It is remarkable that for $\operatorname{ROM}_{(12,5)}^{(c|w / n| a l l, O H)}$ (Figs. 6k and 61) the $\mathrm{OH}$ mass fraction is a little more defined, less spread out near the axis of symmetry, in addition to having a smaller region of a negative mass fraction. The $\mathrm{CH}_{2}$ mass fraction, on the other hand, is a little longer for the ROMs with 5 modes than the ROM using 12 learning cases and 3 modes. Although the length of the $\mathrm{CH}_{2}$ mass fraction for these ROMs is close to the CFD field calculated, the region of maximum concentration is still much smaller. However, for the carbon monoxide the opposite happens, and for $R O M_{(12,5)}^{(u|w| C O)}$, the CO concentration is a little larger than the coupled ROMs.

Comparing the maximum mass fractions value with those calculated by $\mathrm{CFD}$, the predictions made by $R O M_{(12,5)}^{(u|w| p)}$ and $\operatorname{ROM}_{(12,5)}^{(c|w / n| p)}$ are slightly larger than the calculated ones for $\mathrm{OH}$ and $\mathrm{CO}$. For example, the mass fraction found for $\mathrm{CO}$ is 0.053 , whereas the calculated by CFD is 0.051 . Furthermore, the maximum $\mathrm{CH}_{2}$ mass fraction predicted by ROMs is a little lower than the calculated one, $2.8 \cdot 10^{-5}$.

Regarding the effect of increasing number of modes, $R O M_{(12,3)}^{(u|w| p)}$ (Fig. 4) and $R O M_{(12,5)}^{(u|w| p)}$ (Fig. 6), the results for the chemical species are more similar to those calculated by CFD, e.g., in the particular case of $\mathrm{OH}$, the flame front is less spread out and the region of maximum concentration is longer when the ROM uses 5 modes to reconstruct the field. Another example of this effect is the $\mathrm{CH}_{2}$ reconstruction, wherewith the uncoupled ROM using five modes has the description of the flame front longer than the ROM with 3 modes; and the CO field, where the maximum concentration is closer to the calculated by CFD, and also, the region of maximum concentration is longer when compared with the ROM using fewer modes.

All the reduced order models studied here present a white region indicating the presence of negative values for species properties, the only exception is the $\operatorname{ROM}_{(12,3)}^{(u|w| O H)}$, which has a zero minimum value. It is also noticed that for the $\mathrm{CH}_{2}$ reconstruction fields these white areas are more dispersed and without a pattern than the $\mathrm{OH}$ or $\mathrm{CO}$ reconstructions. And this might be caused by the typical $\mathrm{CH}_{2}$ profile, that is, a species with a very narrow concentration field and only present around the flame anchoring region when compared to the others species. In addition to being a minority species in the process, i.e. with a mass concentration on the order of magnitude of $\cdot 10^{-5}$.

\section{CONCLUSIONS}

In this section, the conclusion of the different reduced order models of a laminar diffusion flame are presented.

- The analysis of the amount of data available to construct the reduced order model shows that the number of learning cases has low influence in the energy required. However, the properties reconstruction, mainly the species, show a slight loss, but still preserve the main characteristics.

- It is easier to reproduce the temperature and velocity components than the chemical species, since the first mode has a energy higher than $80 \%$.

- Although the coupling of the properties converges rapidly in the analysis of the energy as a function of the number of modes, e.g., after 3 modes the energy of each mode becomes smaller, less than $0.6 \%$, the results obtained for the chemical species still show error in the description of the flame front, the maximum value and negative mass fraction.

- The normalization of temperature and velocity has little influence on the energy curve, staying very close to the ROM curve without normalization. While the curves of the chemical species have a slightly different behavior, they do not converge as fast. Which may indicate that only the proposed normalization may be insufficient, since some minority species are of the order of magnitude of $10^{-6}$.

- The majority of the reduced order models constructed present some region with negative mass fraction, which is not physically possible. This indicates that the interpolation methods do not preserve the monotonicity, which is paramount for combustion modelling.

\section{ACKNOWLEDGEMENTS}

Louise da C. Ramos developed this project while receiving funding from the European Union's Horizon 2020 research and innovation programme under grant Agreement nr 766264. This work was performed while L.F. Figueira da Silva was on leave from the Institut Prime (CNRS, France). The authors also gratefully acknowledge the support provided by Conselho Nacional de Desenvolvimento Científico e 
Tecnológico, CNPq, under the Research Grants No. 304444/2018-9. This work was financed in part by the Coordenação de Aperfeiçoamento de Pessoal de Nível Superior - Brasil (CAPES) - Finance Code 001.

\section{REFERENCES}

An, J., Wang, H., Liu, B., Luo, K.H., Qin, F. and He, G.Q., 2020. "A deep learning framework for hydrogen-fueled turbulent combustion simulation". International Journal of Hydrogen Energy, Vol. 45, No. 35, pp. 17992-18000. doi:https://doi.org/10.1016/j.ijhydene.2020.04.286.

Ananthkrishnan, N., Deo, S. and Culick, F.E.C., 2005. "Reduced-order modeling and dynamics of nonlinear acoustic waves in a combustion chamber". Combustion Science and Technology, Vol. 177, No. 2, pp. 221-248. doi: https://doi.org/10.1080/00102200590900219.

ANSYS, 2020. "Twin Builder 2021 R1 - Static ROM Components". ANSYS. Twin Bulder Help.

Aversano, G., Ferrarotti, M. and Parente, A., 2021. "Digital twin of a combustion furnace operating in flameless conditions: reduced-order model development from CFD simulations". Proceedings of the Combustion Institute, Vol. 38, No. 4, pp. 5373-5381. doi:https://doi.org/10.1016/j.proci.2020.06.045.

Ben Salem, M., Roustant, O., Gamboa, F. and Tomaso, L., 2017. "Universal prediction distribution for surrogate models". SIAM/ASA Journal on Uncertainty Quantification, Vol. 5, pp. 1086-1109. doi:https://doi.org/10.1137/15M1053529.

Ben Salem, M. and Tomaso, L., 2018. "Automatic selection for general surrogate models". Structural and Multidisciplinary Optimization, Vol. 58, pp. 719-734. doi:https://doi.org/10.1007/s00158-018-1925-3.

Brunton, S.L., Noack, B.R. and Koumoutsakos, P., 2020. "Machine learning for fluid mechanics". Annual Review of Fluid Mechanics, Vol. 52, No. 1, pp. 477-508. doi:https://doi.org/10.1146/annurev-fluid-010719-060214.

Chakravarthy, S.R., Rowan, S.L., Celik, I.B., Gutierrez, A.D. and Escobar Vargas, J., 2015. "A reduced order model for the design of oxy-coal combustion systems". Journal of Combustion, Vol. 2015. doi:https://doi.org/10.1155/2015/943568.

Da Costa Ramos, L., Di Meglio, F., Figueira da Silva, L.F. and Morgenthaler, V., 2020. "Reduced order model of laminar premixed inverted conical flames". In AIAA SciTech Forum. Orlando, USA. doi:https://doi.org/10.2514/6.2020-0416.

De Castro, R.R. and Figueira da Silva, L.F., 2019. "Experimental study of soot volume fraction and temperatue of laminar non-premixed ethylene-air flames". In 25th ABCM International Congress of Mechanical Engineering. Minas Gerais, Brazil.

Gauthier, G.P., Watson, G.M.G. and Bergthorson, J.M., 2012. "An evaluation of numerical models for temperaturestabilized $\mathrm{CH}_{4} /$ air flames in a small channel". Combustion Science and Technology, Vol. 184, No. 6, pp. 850-868. doi:https://doi.org/10.1080/00102202.2012.675376.

Jerez, A., Cruz Villanueva, J., Figueira da Silva, L., Demarco, R. and Fuentes, A., 2019. "Measurements and modeling of pah soot precursors in coflow ethylene/air laminar diffusion flames". Fuel, Vol. 236, pp. 452-460. doi: https://doi.org/10.1016/j.fuel.2018.09.047.

Junqueira, N.L., Figueira da Silva, L. and da Costa Ramos, L., 2020. "Reduced order models applied to laminar diffusion flames". In Proceedings of the 18th Brazilian Congress of Thermal Sciences and Engineering - ENCIT 2020. online.

Kalogirou, S.A., 2003. "Artificial intelligence for the modeling and control of combustion processes: a review". Progress in Energy and Combustion Science, Vol. 29, No. 6, pp. 515-566. doi:https://doi.org/10.1016/S0360-1285(03)00058-3.

Kaymaz, I., 2005. "Application of kriging method to structural reliability problems". Structural Safety, Vol. 27, No. 2, pp. 133-151. ISSN 0167-4730. doi:https://doi.org/10.1016/j.strusafe.2004.09.001.

Kazakov, A. and Frenklach, M., 1984. "Reduced reaction sets based on gri-mech 1.2". The Combustion Laboratory at the University of California, Berkeley. 20 Nov. $2019<$ http://combustion.berkeley.edu/drm/>.

Kieckhefen, P., Pietsch, S., Dosta, M. and Heinrich, S., 2020. "Possibilities and limits of computational fluid dynamics-discrete element method simulations in process engineering: A review of recent advancements and future trends". Annual Review of Chemical and Biomolecular Engineering, Vol. 11, No. 1, pp. 397-422. doi: https://doi.org/10.1146/annurev-chembioeng-110519-075414.

Labahn, J.W., Stanković, I., Devaud, C.B. and Merci, B., 2017. "Comparative study between conditional moment closure (CMC) and conditional source-term estimation (CSE) applied to piloted jet flames". Combustion and Flame, Vol. 181, pp. 172-187. doi:https://doi.org/10.1016/j.combustflame.2017.03.022.

Lancaster, P. and Salkauskas, K., 1981. "Surfaces generated by moving least squares methods". Mathematics of Computation, Vol. 37, No. 155, pp. 141-158. doi:https://doi.org/10.1090/S0025-5718-1981-0616367-1.

Law, C.K., 2006. Combustion Physics. Cambridge University Press, Cambridge.

LeCun, Y., Bengio, Y. and Hinton, G., 2015. "Deep learning". Nature, Vol. 521, p. 436-444. doi: https://doi.org/10.1038/nature14539.

Ostertagová, E., 2012. “Modelling using polynomial regression". Procedia Engineering, Vol. 48, pp. 500-506. doi: https://doi.org/10.1016/j.proeng.2012.09.545.

Peters, N., 2009. "Multiscale combustion and turbulence". Proceedings of the Combustion Institute, Vol. 32, No. 1, pp. 1-25. doi:https://doi.org/10.1016/j.proci.2008.07.044. 
Pyta, L. and Abel, D., 2017. “Online model adaption of reduced order models for fluid flows". IFAC-PapersOnLine, Vol. 50, No. 1, pp. 11138-11143. doi:https://doi.org/10.1016/j.ifacol.2017.08.1006.

Smola, A.J. and Schölkopf, B., 2004. "A tutorial on support vector regression". Statistics and Computing, Vol. 14, p. 199-222. doi:https://doi.org/10.1023/B:STCO.0000035301.49549.88.

Trefethen, L. N.; Bau, D., 1997. Numerical Linear Algebra. Society for Industrial and Applied Mathematics, USA, 1st edition.

Viana, F.A.C., Haftka, R.T. and Steffen, V., 2009. "Multiple surrogates: how cross-validation errors can help us to obtain the best predictor". Structural and Multidisciplinary Optimization, Vol. 39, pp. 439-457. doi: https://doi.org/10.1007/s00158-008-0338-0.

Wang, Q., Hesthaven, J.S. and Ray, D., 2019. "Non-intrusive reduced order modeling of unsteady flows using artificial neural networks with application to a combustion problem”. Journal of Computational Physics, Vol. 384, pp. $289-307$. doi:https://doi.org/10.1016/j.jcp.2019.01.031.

Xiao, X., Fang, F., Buchan, A.G., Pain, C.C., Navon, I.M. and Muggeridge, A., 2015. "Non-intrusive reduced order modelling of the navier-stokes equations". Computer Methods in Applied Mechanics and Engineering, Vol. 293, pp. 522-541. doi:https://doi.org/10.1016/j.cma.2015.05.015.

Zhao, H., 2021. "A reduced order model based on machine learning for numerical analysis: An application to geomechanics". Engineering Applications of Artificial Intelligence, Vol. 100, p. 104194. doi: https://doi.org/10.1016/j.engappai.2021.104194.

\section{RESPONSIBILITY NOTICE}

The authors, Nicole Lopes Junqueira; Luís Fernando Figueira da Silva; Louise da Costa Ramos and Igor Braga de Paula, are solely responsible for the printed material included in this paper. 